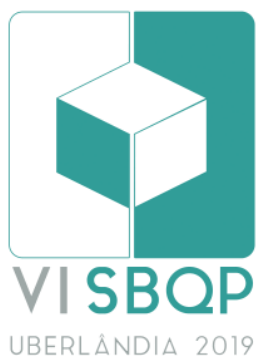

\title{
ANÁLISE DE EFICIÊNCIA ENERGÉTICA UTILIZANDO SOFTWARES BIM: USO DE FERRAMENTAS DE MODELAGEM ENERGÉTICA DO EDIFÍCIO (BEM) DA AUTODESK
}

\author{
OLIVEIRA, Victor \\ Universidade Federal do Espírito Santo - Ufes: e-mail: mrvictormrr@gmail.com \\ JESUS, Luciana \\ Universidade Federal do Espírito Santo - Ufes: Iuciana.njesus@gmail.com \\ CONDE, Karla \\ Universidade Federal do Espírito Santo - Ufes: karlamconde@gmail.com
}

\begin{abstract}
RESUMO
O BIM (Building Information Modeling) surge como um novo paradigma em gerenciamento de projetos seguindo características que o método CAD (Computer Aided Design) disponibilizava de modo dificultado: integração entre as disciplinas, parametrização do projeto, geração instantânea de tabelas, execução de simulações, entre outros. Contudo, apesar dos avanços, o BIM apresenta dificuldades na sua implementação entre algumas interfaces de suas dimensões ou disciplinas. O presente trabalho aborda a problemática da interoperabilidade entre as disciplinas de modelagem, dimensão 3D, e simulação de eficiência energética (BEM - Building Energy Modeling), dimensão 7D, propondo solução a partir da modelagem arquitetônica, realizada no Autodesk Revit® (versão 2019), e a simulação energética do Case600 no ambiente BEM Autodesk [plug-in Insight®, Green Building Studio® e Project Solon®]. Após a simulação, os resultados foram comparados com os outputs de referência obtidos por Queiróz (2016) que utilizou a modelagem e a simulação do Case600 via Sketchup ${ }^{\circledR} 2016$ e EnergyPlus ${ }^{\mathrm{TM}} 8.4 .0$ respectivamente. Constatou-se, que os dispositivos utilizados na pesquisa não atendem aos requisitos de análise estabelecidos pela norma ABNT NBR 15575-1: 2013, verificando assim, ausência de informações nas análises específicas de conforto térmico, como temperaturas internas. No entanto, considerando a sua utilização para análises gerais os mesmos encontramse passíveis de uso.
\end{abstract}

Palavras-chave: BIM, BEM, Eficiência energética, Revit, Insight.

\begin{abstract}
Building Information Modeling (BIM) emerges in construction as a new paradigm in project management to be followed with features that the CAD (Computer Aided Design) method offers in a difficult way: integration among disciplines, project parameterization, instant generation of tables, execution of simulations etc. However, despite the advances, BIM presents difficulties in its implementation between some interfaces of its dimensions or disciplines. The present paper addresses the interoperability problem between the disciplines of architectural modeling, 3D dimension, and simulation of energy efficiency (BEM), dimension 6D, proposing a solution based on modeling, performed in Autodesk Revit® (2019), and the energy simulation of the Case600 in the BEM Autodesk environment [Insight $\AA$ Devices, Green Building Studio ${ }^{\circledR}$ and Project Solon $\AA$ ]. After the simulation, the results were compared with the reference results obtained by Queiróz (2016) who used the modeling and simulation of the Case600 via Sketchup \& 2016 and EnergyPlus ${ }^{\mathrm{TM}}$ 8.4.0, respectively. It was verified that the devices used in the research did not meet the analysis requirements established by ABNT NBR 15575-1: 2013, thus verifying the miss information in the specific analysis of thermal comfort, such as internal temperatures. However, for general analyzes able to be used.
\end{abstract}

Keywords: BIM, BEM, Energy Efficiency, Revit, Insight.

OLIVEIRA, V.; JESUS, L.; CONDE, K. Análise de eficiência energética utilizando softwares BIM: Uso de ferramentas de modelagem energética do edifício (BEM) da Autodesk. In: SIMPÓSIO BRASILEIRO DE QUALIDADE DO PROJETO NO AMBIENTE CONSTRUÍDO, 6., 2019, Uberlândia. Anais... Uberlândia: PPGAU/FAUeD/UFU, 2019. p. 943-954. DOI https://doi.org/10.14393/sbqp19087. 


\section{INTRODUÇÃO}

Os avanços tecnológicos do fim do século XX proporcionaram para diversos segmentos da indústria mundial a otimização dos seus processos. Esses avanços evidenciaram-se na construção civil a partir do ano 2000, quando métodos como o Building Information Modeling (BIM) tornaram a execução de projetos informatizada, integrada, parametrizada, visualmente acessível (3D), otimizada (comparação ao método CAD (Computer Aided Design)) entre outras vantagens (CBIC, 2016).

O BIM é particionado em várias dimensões de trabalho as quais abrangem todo o processo produtivo de um empreendimento e são denominadas " $\mathrm{nD}$ ", isto é, 3D, 4D, 5D, 6D, 7D etc. Logo, no processo de implementação, notou-se a necessidade de interoperabilidade entre essas dimensões de modo que o BIM mantivesse suas características de otimização e integração (ABDI, 2017).

Uma problemática quanto a interoperabilidade se dá entre a dimensão 3D, modelagem do projeto, e $\bigcirc$ 7D, análise de eficiência energética ou denominada como BEM (Building Energy Modeling).

Os softwares de modelagem BIM usuais (3D) não possuem interoperabilidade confiável e eficaz com o EnergyPlus ${ }^{T M}$, software BEM (7D) referência em desempenho energético (QUEIRÓZ,2016) (CBIC,2016).

Esse caso particular acontece, por exemplo, entre o software Autodesk Revit $M E P^{\circledR}$ e $\circ$ EnergyPlus ${ }^{\text {TM }}$, os quais não conseguem estabelecer um ambiente eficaz para análise de eficiência energética.

Logo, as Ferramentas (BEM) Autodesk de análise de energia: Insight ${ }^{\circledR}$, Green Building Studio ${ }^{\circledR}$ e Project Solon ${ }^{\circledR}$ posicionam-se como uma alternativa para sanar a ausência interoperabilidade entre o Revit $^{\oplus}$ e o EnergyPlus ${ }^{\mathrm{TM}}$.

A partir desse panorama, o intuito de parte deste projeto de pesquisa, é investigar por meio da bibliografia disponível e por meio da simulação do Case600 no ambiente BEM Autodesk se essas ferramentas geram resultados confiáveis de desempenho térmico e energético de uma edificação a partir de um teste comparativo com dados de referência apresentados por QUEIRÓz (2016) que foram gerados pelo EnergyPlus ${ }^{\mathrm{TM}}$ 8.4.0.

\section{REVISÃO BIBLIOGRÁFICA}

\subsection{Fluxo de trabalho BIM}

Devido a ampla aplicabilidade do BIM ao ciclo de vida do empreendimento, seja em processos de modelagem dos projetos à processos de orçamentação, manutenção e demolição, por exemplo, autores como Eastman e Karmeedan definiram os usos do BIM em "nD" dimensões, sendo cada dimensão um fluxo de trabalho dentro do ciclo de vida do empreendimento. A interoperabilidades entre essas dimensões compõe o plano de colaboração BIM (ABDI, 2017 Vol 3; QUEIRÓZ, 2016).

A dimensão 3D relaciona-se a modelagem do projeto como um todo, não apenas uma única disciplina, representando os parâmetros e dados de cada objeto e elemento construtivo (BRACHT, 2016; ABDI, $2017 \mathrm{Vol} 1$ ).

$\mathrm{O}$ 4D é relativo ao planejamento de obra. Com a modelagem advinda do 3D simula-se as atividades de canteiro de obra por meio de cronogramas 
visualizando em animações 3D toda a sequência de execução da obra (BRACHT, 2016; ABDI, 2017 Vol 1).

Após a modelagem e o planejamento de obra a extração de quantitativos torna-se eficiente, pois ao modelo está associado um banco de dados. O 5D é - processo que remete ao custo do projeto, geração instantânea de orçamentos e dados financeiros baseados nos quantitativos dos elementos construtivos e objetos paramétricos (BRACHT, 2016; ABDI, $2017 \mathrm{Vol} 1$ ).

A dimensão 6D é referente aos pós obra. Devido aos objetos serem parametrizados é possível incluir dados de manutenção e gerenciamento de obra, com isso, softwares conseguem acessar o banco de dados do modelo e emitir alertas de manutenção, por exemplo (BRACHT, 2016; ABDI, $2017 \mathrm{Vol} 1$ ).

O 7D remete a sustentabilidade e eficiência energética do projeto. Atua em simulações energéticas, térmicas e acústicas com base nas propriedades dos materiais e na conformação do empreendimento. Identificando por meio das simulações alternativas mais eficientes em questões de conforto e custo (ABDI, 2017, Vol 5).

O 8D incorpora aspectos de segurança, tanto na elaboração do projeto quanto no decorrer das atividades de construção. As dimensões, por mais que aqui sejam descritas do 3D ao 8D adquirem caráter infinito devido a não limitação da aplicabilidade do BIM, sendo as dimensões artifícios que organizam a atuação de cada plano de trabalho (ABDI, $2017 \mathrm{Vol} 1$ ).

Apesar das definições formais, muitos autores não se atêm a definir o que é BIM, pois de certa forma a definição torna-se burocrática e difícil ao entendimento. Logo, eles preferem definir o que não é BIM (CBIC, 2016).

Portanto, lança-se mão do mesmo artifício e se sequencia algumas características que não são oriundas do BIM (CBIC, 2016):

1. Soluções 3D ausentes de gestão de banco de dados de informações dos objetos e do projeto;

2. Ausência de funcionalidades que permitem extração de quantidades do projeto a qualquer instante;

3. Objetos não paramétricos;

4. Atualizações não automáticas;

5. Ausência de gestão de banco de dados.

Por esses motivos, a mudança do modelo CAD para o Building Information Modeling possibilita que projetistas dispendam mais tempo projetando do que se atendo a processos de documentação e outras atividades manuais e de conhecimento tácito as quais são automatizados, de maneira instantânea, no novo plano de trabalho (BRACHT, 2016).

\subsection{Interoperabilidade como estigma do BIM}

Frente a abrangência da nova metodologia, evidencia-se um obstáculo que deve ser ultrapassado: a ausência de interoperabilidade.

A interoperabilidade possuí relevância considerável para o desenvolvimento em BIM de modo que se torna também um ponto crítico, pois se os softwares 
BIM não se comunicam não há fluxo de trabalho BIM voltando desta forma às características arcaicas e pouco produtivas, comuns ao CAD (BRACHT, 2016).

Essa problemática é evidente na dimensão 7D em que softwares BIM consagrados no mercado em diversas disciplinas e dimensões BIM não possuem desempenho satisfatório nas simulações de eficiência energética de modo que também não propiciam exportação eficiente para softwares de simulação que são referência em análise de energia. Portanto, nota-se que há soluções, contudo elas não se comunicam, não são compatíveis no momento de migrar os dados de uma dimensão BIM para outra (QUEIROZ, 2016).

Diante disso, usuários utilizam softwares como o Sketchup ${ }^{\circledR}$, da desenvolvedora Trimble, aliado ao plugin Euclid, da desenvolvedora BigLadder (que confere propriedades térmicas aos itens modelados), (ambos não são softwares BIM ou BEM), para remodelar o projeto arquitetônico, por exemplo, a fim de exportálo para o software de referência em análise de eficiência energética, Energy Plus, recomendado tanto por normas brasileiras, NR15575-1 - Edificações Habitacionais - Desempenho Parte 1: Requisitos gerais, quanto por normas americanas, ASHRAE (lAmerican Society of Heating, Refrigerating and Air Conditioning Engineers) Standard 140.

Essa atitude de remodelagem do projeto configura-se como um retrabalho contrariando veemente o conceito BIM (ABNT, 2013) (QUEIROZ, 2016).

\subsection{Soluções aparentes a problemática da interoperabilidade no 7D}

Na construção civil o software Autodesk Revit ${ }^{\circledR}$ destaca-se como líder de mercado e como a ferramenta BIM mais difundida principalmente no Brasil. $O$ Revit aborda as disciplinas Architeture, MEP(mechanical, electrical, and plumbing) e Structure dando vazão ao plano de trabalho em um mesmo software. Além de possuir integração e plena interoperabilidade com vários outros softwares Autodesk como AutoCad Civil 3D ${ }^{\circledR}$, Navisworks ${ }^{\circledR}$, Robot $^{\circledR}$, Insight ${ }^{\circledR}$ etc. Os quais aprofundam as outras dimensões BIM (QUEIRÓZ, 2016) (BRACHT, 2016).

Apesar do forte posicionamento do Autodesk Revit no segmento de modelagem ele não apresenta uma interoperabilidade eficiente e confiável com o EnergyPlus ${ }^{\mathrm{TM}}$, como apontou QUEIRÓZ (2016). Os arquivos exportados em .idf (extensão do EnergyPlus ${ }^{\mathrm{TM}}$ ) não levam todos os parâmetros e dados de materiais inseridos na modelagem no software da Autodesk, gerando distorções no modelo final e com isso demandando vários ajustes, retrabalhos e consequentemente falhas nas simulações.

A Autodesk, porém, desenvolveu ao longo dos anos softwares e dispositivos que a partir dos mesmos mecanismos de cálculo do Energyplus ${ }^{\mathrm{TM}}$ proporcionam ao fluxo de trabalho, a partir do Revit ${ }^{\circledR}$, a possibilidade de simulações de eficiência energética. Os softwares para tal fim são, o mais recente, Autodesk Insight e as versões antecessoras a ele: Green Building Studio ${ }^{\circledR}$ e Project Solon ${ }^{\circledR}$, todos disponíveis na nuvem.

A IBPSA (International Building Performance Simulation Association) foi convidada pelo Departamento de Energia dos Estados Unidos (Fomentadores do Energyplus ${ }^{\mathrm{TM}}$ ) a gerenciar o diretório da Web Building Energy Simulation Tools (BEST-D). Em seu site a IBPSA classifica o Insight e o Energyplus ${ }^{\mathrm{TM}}$ pelos seguintes itens da Tabela 1 (IBPSA, 2019). 
Tabela 1 Descrição das atribuições do Insight e Energyplus ${ }^{\mathrm{TM}}$ pela IBPSA

\begin{tabular}{|c|c|}
\hline Autodesk Insight & Energyplus $^{\mathrm{TM}}$ \\
\hline Simulação de todo o empreendimento & Simulação de todo o empreendimento \\
\hline Parâmetros e otimização & $\begin{array}{c}\text { Seleção e dimensionamento de sistema } \\
\text { HVAC }\end{array}$ \\
\hline Simulação de lluminação & Simulação de iluminação \\
\hline Análise Solar e Fotovoltaica & Simulação de fluxo de ar \\
\hline & Cálculo de Cargas térmicas \\
\hline
\end{tabular}

Fonte: IBPSA (2019), modificado pelos autores

A partir da Tabela 1 nota-se que o Autodesk Insight ${ }^{\circledR} 2019$ se equipara, em teoria, ao EnergyPlus ${ }^{\mathrm{TM}}$ 8.4.0. E, portanto, seguindo uma alternativa à linha de pesquisa de QUEIRÓz (2016) o trabalho se propõe a investigar se as ferramentas: Autodesk Insight ${ }^{\circledR}$, Green Building Studio ${ }^{\circledR}$ e o Project Solon ${ }^{\circledR}$ conseguem gerar outputs compatíveis com os dados do Energyplus ${ }^{\mathrm{TM}}$ 8.4.0 (Temperaturas mensais anuais internas) obtidos por QUEIRÓZ (2016) a partir do CASE 600, um modelo específico apresentado pela ASHREA Standard 140 e exibido na Figura 1.

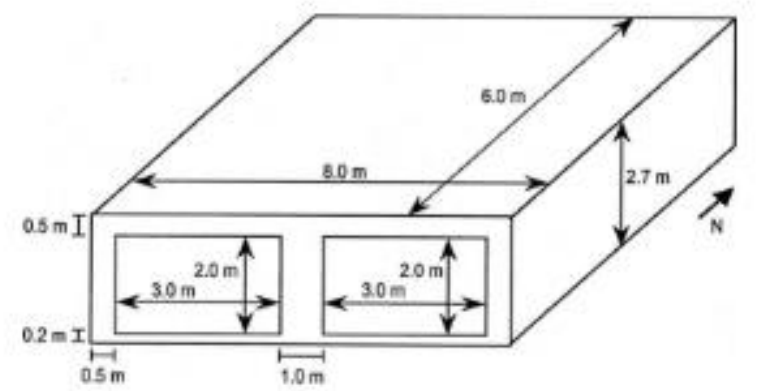

Figura 1 - Case 600 e suas dimensões -

Fonte: ASHREA Standard 140 apud QUEIROZ (2016)

\subsection{Ferramentas Autodesk BEM}

O conjunto de softwares ou plug-in Autodesk BEM são, pode-se dizer, a evolução da análise dos dados para uma interface gráfica atrativa ao usuário. Todas as três ferramentas possuem mecanismos e métodos de exibição que facilitam a exposição e manipulação dos dados.
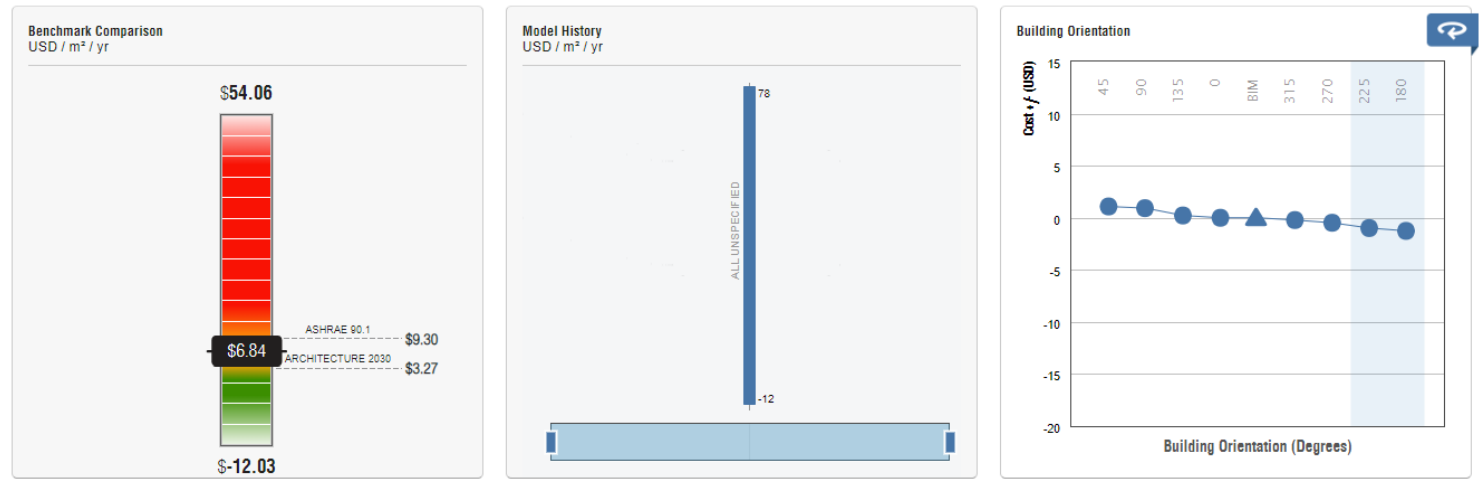

Figura 2 - Widgets presentes no Insight -

Fonte: Insight (2019) 
O Insight coloca-se como uma evolução do Green Building Studio e Project Solon. Hoje, é o software recomendado pela Autodesk para análise de eficiência energética. Contudo, os antecessores são ainda passíveis de uso, pois o Insight não possui todos as funções de ambos.

A Figura 2 apresenta a interface do Insight em que ao alterar ou mover os pontos dos mecanismos exibidos eles automaticamente recalculam os custos anuais de consumo, taxas de infiltração e outros parâmetros de análise.

\section{METODOLOGIA}

Por meio da revisão bibliográfica identificou-se entre os trabalhos e autores a dissertação desenvolvida por QUEIRÓz (2016) como uma base de dados de referência para efeito de comparação com os dados gerados pelos dispositivos Insight $\AA^{\circledR}$, Green Building Studio ${ }^{\circledR}$ e Project Solon ${ }^{\circledR}$ versões disponíveis em 2019 que receberam via cloud [online] o projeto exportado pelo Autodesk Revit Architecture® versão 2019.

QUEIRÓZ (2016) elaborou os dados de referência modelando o Case600 por meio do Sketchup ${ }^{\circledR} 2016$ e o plugin Open Studio ${ }^{\circledR}$ (versão 1.0.14) o qual exportou o modelo para simulação no EnergyPlus ${ }^{\text {8Wa }} 8.4 .0$ obtendo outputs de temperatura interna média em ${ }^{\circ} \mathrm{C}$ que foram usados como referência.

Logo, tomaram-se esses dados de modelagem e realizou-se um caminho alternativo: modelagem no çRevit ${ }^{\circledR} 2019$, exportação para o ambiente BEM Autodesk, geração de resultados e comparação com os resultados referência de Queiróz (2016).

Desse modo, tomou-se o Autodesk Revit ${ }^{\circledR} 2019$ a fim de modelar o Case600 seguindo os dados de construção e materiais disponibilizados por QUEIRÓz (2016) e utilizados pelo mesmo. Executou-se e se detalhou todas as configurações de energia presentes e disponíveis no programa de modelagem. Segundo AUTODESK (2018) e QUEIRÓZ (2016) a definição desses parâmetros possuem um check-list particular. A seguir estão as etapas e itens cumpridos na configuração do modelo para um satisfatório processo de análise:

1) Modelar o projeto do empreendimento a ser analisado atentando-se a inserção dos materiais e seus respectivos dados aos elementos construtivos;

2) Adicionar espaços e zonas. Lembrando que os espaços a serem levados em conta devem ser adicionados em zonas diferentes da zona padrão;

3) Especificar as configurações de área e volume;

4) Configurar tipos de construção e espaço;

5) Especificar a localização do empreendimento;

6) Configurar cargas de aquecimento e resfriamento

a. Geral;

b. Detalhes;

7) Configurar os itens de configuração de energia. 
A Figura 3 exibe o menu analisar do $\operatorname{Revit}^{\circledR}$ em que há os principais itens de configuração de energia do modelo. Na guia Relatórios e tabelas configuramse as cargas de aquecimento e resfriamento, como configuração de zonas, espaços e inserção de sistemas HVAC; Já na guia Otimização de energia encontram-se os ícones de localização, geração de modelo de energia, otimização do modelo e configuração de energia, este último é onde configura-se os modos de exportação e outras configurações gerais de energia.

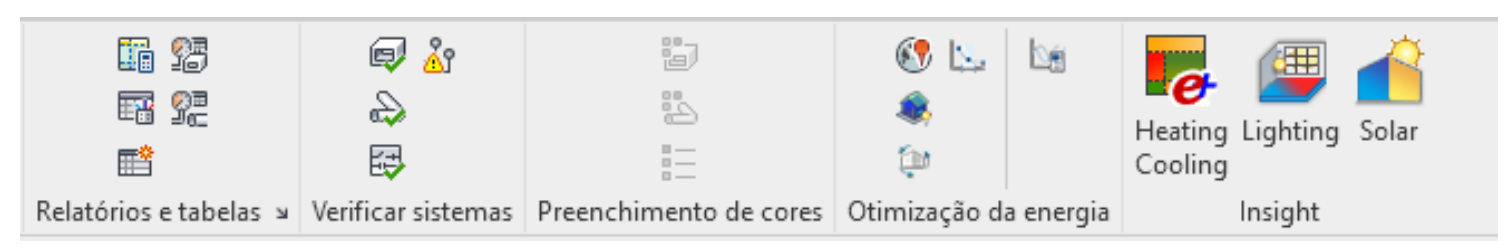

Figura 3 - Modelo BIM do Case600 -

Fonte: Revit (2019)

Todos as configurações, inputs, dos itens do check-list acima foram coletados de QUEIROZ (2016) a fim de manter a base comparativa com os dados extraídos do trabalho de referência.

Por fim, gerou-se um modelo de energia, em seguida exportou-se o modelo de energia gerado para o ambiente BEM Autodesk online, realizando, assim, a simulação do modelo BIM. Então, com os resultados, efetuou-se o exercício de comparação com os valores de referência. A sequência de atividades descritas está exemplificada por meio do fluxograma da Figura 4.

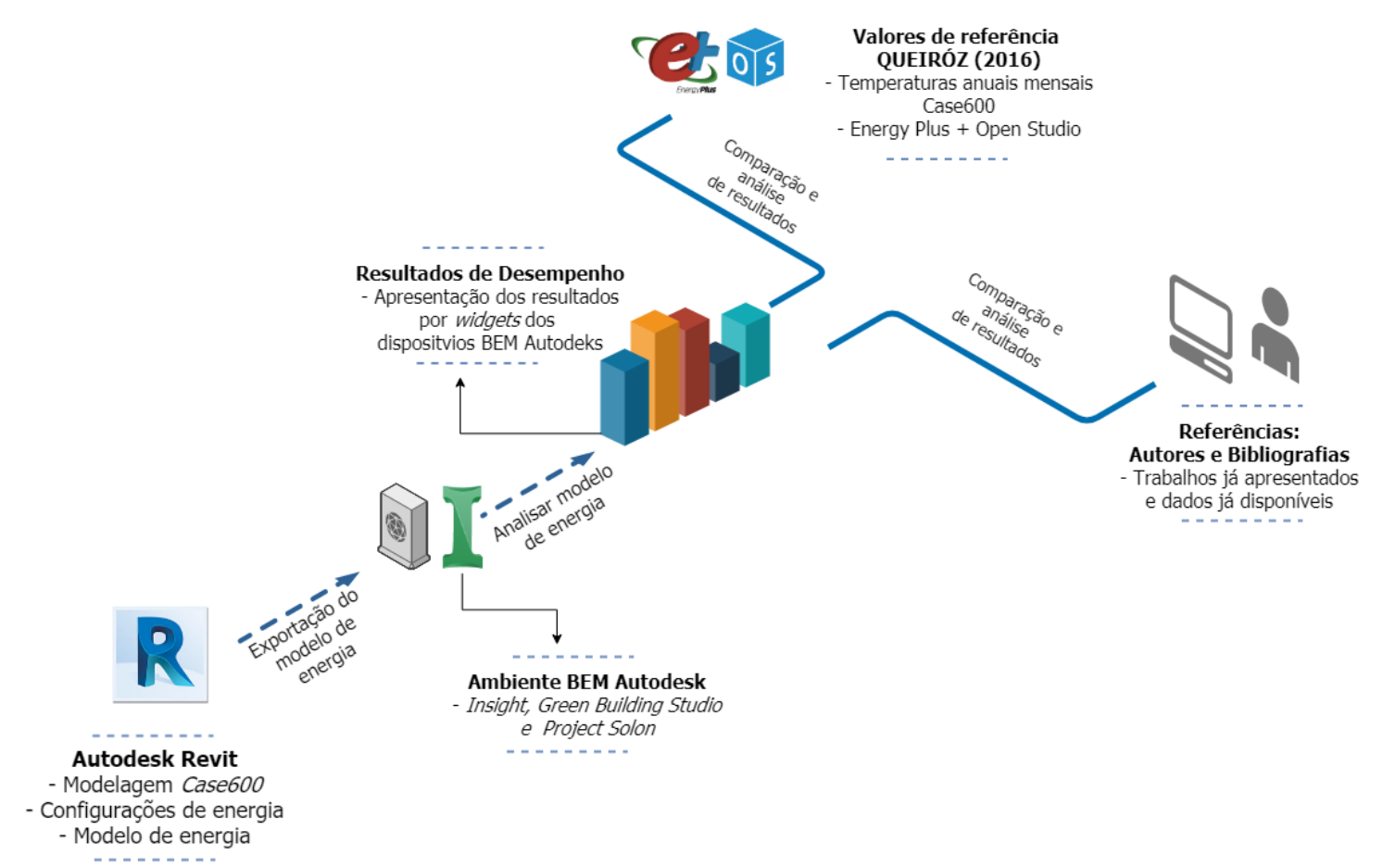

Figura 4 - Metodologia de análise exemplificada por meio de dois segmentos Fonte: Elaborado pelo autor (2019) 
Findada todas as configurações descritas e a consequente geração do modelo de energia, foram realizadas as análises de energia, cargas de aquecimento e resfriamento disponíveis pelo Revit ${ }^{\circledR}$ e pela nuvem do sistema Autodesk. Logo, verificaram-se as seguintes possibilidades de simulação:

1) Cálculo de Cargas de Aquecimento e resfriamento;

2) Otimização do modelo na nuvem (Insight ${ }^{\circledR}$, Green Building Studio ${ }^{\circledR} e$ Project Solon $\left.{ }^{\circledR}\right)$;

3) Lighting;

4) Solar.

\section{RESULTADOS E DISCUSSÃO}

Com as especificações construtivas, valores das propriedades dos materiais e dimensões do modelo Case600 efetuou-se o exercício de modelagem no Autodesk Revit MEP ${ }^{\circledR}$. Obtendo-se, então, o modelo exibido na Figura 5.

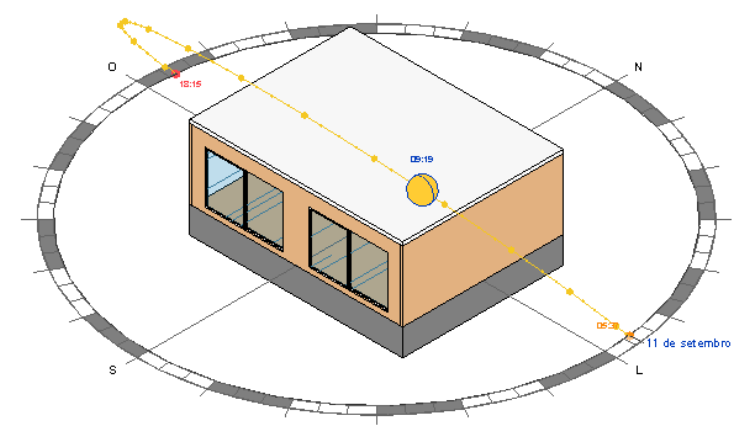

Figura 5 - Modelo BIM do Case 600 -

Fonte: Elaborado pelo autor com base em Queiróz (2016) e com auxilio do Revit 2019 (2019)

Após a modelagem detalhou-se e se executou as configurações de energia necessárias para a geração do modelo de energia seguindo o check-list sugerido.

Feitas as configurações o modelo BIM gerou-se e se otimizou o modelo de energia na cloud, isto é, ao acionar essas opções o modelo foi enviado para o ambiente online BEM Autodesk, ou seja, o modelo foi disponibilizado para análise do Insight ${ }^{\circledR}, G B S^{\circledR}$ e Project Solon ${ }^{\circledR}$. Essa interface geral é exibida na Figura 6. Também, ainda na interface do Revit ${ }^{\circledR}$, acionou-se as outras possibilidades de análise: Cálculo de cargas de aquecimento e resfriamento; Heating Cooling; Lighting; Solar.

\section{AUTODESK}

GREEN BUILDING STUDIO॰

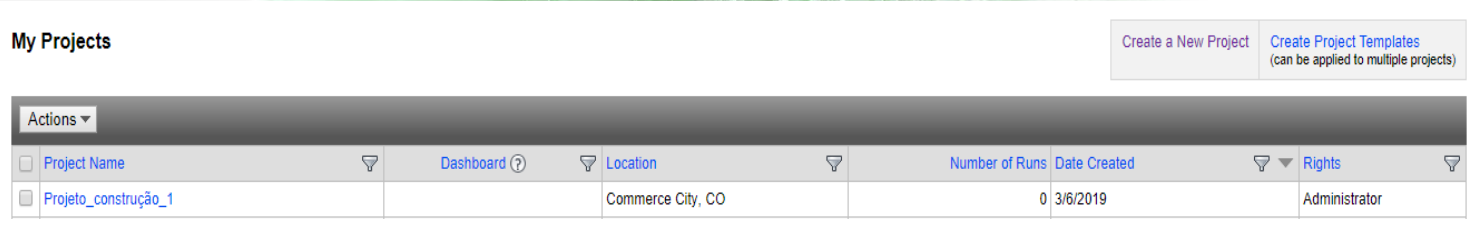

Figura 6 - Ambiente BEM Autodesk -

Fonte: Ambiente na nuvem Green Building Studio (2019) 


\subsection{Cálculo de cargas de aquecimento e resfriamento}

Nessa ferramenta é gerado um relatório com dados anvais que se referem as trocas e influências térmicas dos elementos presentes na construção e nos ambientes. Informações como: perfil de uso, iluminação, e os materiais dos elementos construtivos, foram mantidos conforme trabalho apresentado por QUEIRÓZ (2016).

A Figura 7 apresenta uma amostra de uma parte do relatório, com valores correspondentes a uma análise anual das trocas de carga. Essa opção de simulação é pouco customizável, não permitindo, por exemplo, a mudança do período de simulação anual para outro período, como um mês do ano em específico. Contudo, apresenta valores de trocas de calor que ocorrem por meio de elementos construtivos e devido a ocupação das pessoas no ambiente além de outros dados como pode ser visto na figura indicada.

Resumo da Construção
\begin{tabular}{|l|l}
\hline Entradas & Unifamiliar \\
\hline Tipo de construção & 48 \\
\hline Área $\left(\mathrm{m}^{2}\right)$ & 129.60 \\
\hline Volume $\left(\mathrm{m}^{\mathrm{s}}\right)$ & \\
\hline Resultados calculados & 6,494 \\
\hline Carga total de pico de resfriamento $(\mathrm{W})$ & Setembro 13:00 \\
\hline Mês e hora do piso de resfriamento & 7,119 \\
\hline Carga sensivel do pico de resfriamento $(\mathrm{W})$ & -625 \\
\hline Carga latente do pico de resfriamento $(\mathrm{W})$ & 6,323 \\
\hline Capacidade máxima de resfriamento $(\mathrm{W})$ & 453.7 \\
\hline Fluxo de ar do pico de resfriamento $(\mathrm{L} / \mathrm{s})$ & 3,970 \\
\hline Carga do pico de aquecimento $(\mathrm{W})$ & 327.7 \\
\hline Fluxo de ar do pico de aquecimento $(\mathrm{L} / \mathrm{s})$ & \\
\hline Verificação de somas & 135.29 \\
\hline Densidade da carga de resfriamento $\left(\mathrm{W} / \mathrm{m}^{2}\right)$ & 9.45 \\
\hline Densidade do fluxo de resfriamento $\left(\mathrm{L} /\left(\mathrm{s} \cdot \mathrm{m}^{2}\right)\right)$ & 69.87 \\
\hline Fluxo / Carga de resfriamento $(\mathrm{L} /(\mathrm{s} \cdot \mathrm{kW}))$ & 7.39 \\
\hline Área/ Carga de resfriamento $\left(\mathrm{m}^{2} / \mathrm{kW}\right)$ & 82.70 \\
\hline Densidade da carga de aquecimento $\left(\mathrm{W} / \mathrm{m}^{2}\right)$ & 6.83 \\
\hline Densidade do fluxo de aquecimento $\left(\mathrm{L} /\left(\mathrm{s} \cdot \mathrm{m}^{2}\right)\right)$ & \\
\hline
\end{tabular}

Figura 7 - Relatório de Cargas de aquecimento e resfriamento -

Fonte: Elaborado pelo autor com auxílio do Revit (2019)

Nota-se, por meio da análise dos valores gerados que se referem a taxas de desempenho e troca de cargas, não apresentando informações de temperaturas internas médias dos ambientes ou algo que fosse similar as saídas apresentadas pelo trabalho de referência.

\subsection{Lighting e Solar}

Essas duas funções são de certa forma complementares, o que as distingue é o ambiente em que são executadas e a natureza dos dados gerados. A função Solar permite que o usuário, visualmente, interprete o grau de incidência solar sobre superfícies previamente selecionadas. Com isso, podese, de forma facilitada reavaliar o modelo e a envoltória de acordo com o grau de incidência, assim como prever anteparos a fim de gerar sombras. A Figura 8 apresenta os resultados obtidos por meio dessa ferramenta.

Já a função Lighting é executada na nuvem por meio de créditos pagos e fornece análises de iluminação. Pode ser considerada complementar à função Solar, pois esta permite também identificar o grau de incidência solar 
nos ambientes que complementa a ferramenta exibida na Figura 9. Essa ferramenta não foi utilizada neste trabalho.

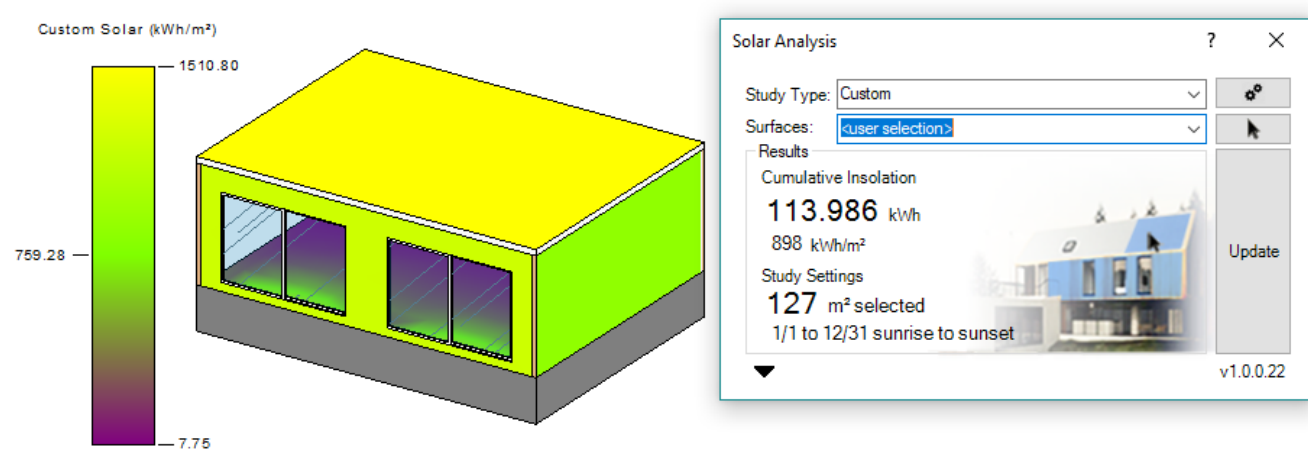

Project location: E 58th Ave, Commerce City, Co 80022, Estados Unidos

Sun study start date time: 0 1//01/2010 00:00:00
Sun study end date time: $31 / 12 / 2010$ 23:59:00

Cumulative Insolation

Figura 8 - Insolação sobre superfícies do modelo CASE600 -

Fonte: Elaborado pelo autor com auxílio do Revit (2019)



$\square$ Email: mrvictormrr when complete

$\vee 3.1 .0 .5$

Start Analysis Cancel

Figura 9 - Análise de eficiência luminosa ou lumínica na nuvem por meio da ferramenta Lighting -

Fonte: Elaborado pelo autor com auxílio do Revit (2019)

Ambas alternativas não fornecem resultados para fins comparativos com o trabalho referência desse artigo, contudo, são citadas para exposição das possibilidades que o Revit proporciona para a análise de eficiência energética, por mais que elas não são efetivas, por ora. 


\subsection{Insight, Green Building Studio e Project Solon}

Após envio do modelo para nuvem, tomou-se os resultados das simulações gerados pelas três ferramentas visando a obtenção de índices de temperatura interna média para estabelecer uma base comparativa.

Entretanto, a exemplo dos resultados obtidos por meio do Revit (Ferramentas exibidas anteriormente), sem o auxílio da nuvem, nenhum destes dispositivos BEM possuíam mecanismos de cálculo capazes de simular e apresentar os dados em intervalos de tempo como o EnergyPlus ${ }^{\mathrm{TM}}$, isto é, essas ferramentas não exibiram, por exemplo, temperaturas internas de ambientes ou superfícies em intervalos de horas, dias, semanas ou meses, mas apenas dados anuais.

Segundo a ABNT NBR 15575-1:2013, são definidos requisitos para validação de resultados de análise de eficiência energética e desempenho térmico, sendo o principal deles, o fornecimento de dados relacionados as temperaturas médias mensais internas dos espaços e zonas do modelo em análise. Os dados de referência do Case600 obtidos por Queiróz (2016) seguem essa prerrogativa, o que já não foi observado pelos resultados dos dispositivos Autodesk.

O mais próximo que se consegue são temperaturas de bulbo seco a qual é a temperatura do ar medida por um termômetro com dispositivo de proteção

contra a influência da radiação térmica. Esse resultado, ainda, foi obtido e proporcionado somente pelo dispositivo Project Solon ${ }^{\circledR}$, uma versão anterior ao Insight ${ }^{\circledR}$ (ABNT,2003).

Se por um lado constata-se a ausência de parâmetros relevantes, como temperatura do ambiente interno, por outro, vale ressaltar que outputs dos dispositivos BEM Autodesk são passíveis de comparação e uso. Conclui-se que os outputs possuem um enfoque maior no desempenho energético da edificação, não abordando satisfatoriamente dados de conforto térmico, por exemplo.

Outputs como melhor orientação da edificação, estudos de iluminação e incidência solar, analise de rentabilidade de sistema fotovoltaico entre outros ao serem exibidos podem ser manipulados de forma a se buscar um menor consumo energético anual. O resultado das alterações e escolhas de novas alternativas, como uma melhor orientação da edificação instantaneamente alteram o consumo anual.

Contudo, como a base de referência escolhida apenas gerou outputs mensais de temperatura ao longo do ano, além de outros fatores já explicitados, não se torna útil para validar as outras saídas BEM da Autodesk.

VENDRAME (2017), em seu estudo de caso, por exemplo, mostra que apesar das limitações, os outputs BEM Autodesk possibilitam estabelecer um diagnóstico factível e passível de sugerir medidas para melhor eficiência energética da edificação.

\section{CONSIDERAÇÕES FINAIS}

Os dispositivos de análise BEM Autodesk não apresentaram resultados satisfatórios quanto a exigência normativa NBR 15575:2013 acerca de outputs de temperaturas médias mensais internas, contudo, os outros resultados apresentados, acerca de desempenho térmico e energético, são passíveis, 
apesar de suas limitações, de uso para fins de diagnóstico energético de empreendimentos e para a tomada de decisão na etapa de desenvolvimento do projeto.

Ressalta-se também a importância de trabalhos como o de QUEIROZ (2016) o qual por meio de sua metodologia propiciou a análise da efetividade do Revit 2019 e seus dispositivos no que tange a análise de eficiência energética. E a partir desses resultados propõe-se uma linha de pesquisa que vise utilizar as ferramentas BEM Autodesk e outros softwares BIM, buscando não mais índices relativos a temperaturas internas médias, mas conferindo enfoque aos resultados de desempenho disponíveis na plataforma.

\section{REFERÊNCIAS}

ASSOCIAÇÃO BRASILEIRA DE NORMAS TÉCNICAS. NBR 15575-1: Edificações Habitacionais - Desempenho. Parte 1: Requisitos gerais para validação de resultados de análise de eficiência energética e desempenho térmico, 2013.

NBR 15220-1: Desempenho térmico de edificações Parte 1:

Definições, símbolos e unidades, 2003.

AGÊNCIA BRASILEIRA DE DESENVOLVIMENTO INDUSTRIAL (ABDI). Processo de Projeto BIM: Coletânea Guias BIM ABDI-MDIC / Agência Brasileira de Desenvolvimento Industrial. -Brasília, DF: ABDI, 2017. Vol. 1, 22 p.

Avaliação de desempenho energético em Projetos BIM: Coletânea

Guias BIM ABDI-MDIC / Agência Brasileira de Desenvolvimento Industrial. Brasília, DF: ABDI, 2017. Vol. 5; 22.p

CBIC. Fundamentos BIM - Parte 1: Implementação do BIM para Construtoras e Incorporadoras/Câmara Brasileira da Indústria da Construção. - Brasília: CBIC, 2016. 124p.

QUEIRÓZ, G. R. Análise de Interoperabilidade entre os programas computacionais Autodesk Revit e Energy Plus para a simulação térmica de edificações. 169 p. Dissertação (Mestrado) - Universidade Federal de Santa Maria, 2016.

VENDRAME, L. G. V. OTIMIZAÇÃO ENERGÉTICA NUM SUPERMERCADO UTILIZANDO O REVIT® E ESTRATÉGIAS DE DAYLIGHTING - SOLUÇÕES PASSIVAS E ATIVAS. Instituto Superior de Engenharia do Porto: Departamento de Engenharia Mecânica, 2017. 